\title{
Transcatheter Aortic Valve Implantation: Does General Anesthesia reduce the incidence of postprocedural aortic regurgitation compared to Sedation?
}

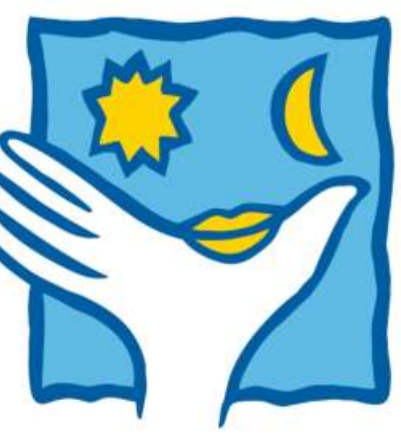

CHU

Hôpitaux de Bordeaux

Cedrick ZAOUTER*, L. Leroux, L. Labrousse, G. Bonnet,S. Lafitte, A. Ouattara

*Service d'Anesthésie-réanimation II,

Haut-Lévêque hospital, CHU Bordeaux, France.

Email: cedrick.zaouter@chu-bordeaux.fr

\section{Introduction}

Transcatheter aortic valve implantation (TAVI) is a valid option for patients with severe aortic stenosis judged to be at a high surgical risk.

There is still no agreement regarding the appropriate type of anesthesia.

If sedation presents several advantages, it has been suggested that general anesthesia lead to less paravalvular leaks (PVL) likely because of the transesophageal echocardiography (TEE) guidance ${ }^{1}$.

\section{Study Objective}

To compare the incidence of PVL between patients receiving sedation (TAVI-S) and patients receiving general anesthesia (TAVI-GA).

\section{Methods}

Patient's characteristics, the incidence and grade of PVL were collected in patients undergoing TAVI.

The TAVI-S group, received local anesthesia and a continuous infusion of a sedative agent.

The TAVI-GA group, received a total intravenous anesthesia to allow the insertion of an endotracheal tube and a TEE probe.

-After the implantation, PVL were deemed by hemodynamic and fluoroscopic measurements in the TAVI-S group. TEE was also used in the TAVI-GA group.

-A post-dilatation was performed when PVL were $>$ than mild.

-PVL was assessed with transthoracic echocardiography in both groups on postop day 5 .

\section{Results}

TAVI-S and TAVI-GA was accomplished in $\mathbf{1 6 8}(\mathbf{6 7 . 5 \%})$ and in $81(32.5 \%)$ patients, respectively.

The two groups were similar except for a more frequent history of coronary artery disease $(\mathrm{p}=0.004)$, peripheral artery disease $(\mathrm{p}<0.001)$ and diabetes $(\mathrm{p}=0.028)$ in the TAVI-GA group. Six patients died during the procedure.

Table 1 depicts the incidence and grade of PVL.

\section{Conclusions}

Our study indicates that, in an experienced center, moderate to severe $P V L$ is rare and TAVI-GA with TEE guidance doesn't reduce it.

Table 1: Incidence and grade of paravalvular leaks

After valve deployment

Absent to mild, $\mathrm{n}(\%)$

TAVI- $S(n=165)$

Moderate to severe, $\mathrm{n}(\%)$

159(96)

On postoperative day 5

$6(4)$

Absent to mild, $\mathrm{n}(\%)$

TAVI-S $(n=163)$

Moderate to severe, $\mathrm{n}(\%)$

159(98)

$4(2)$

\begin{tabular}{|l||l}
\hline TAVI-GA $(n=78)$ & P-Value \\
\hline $76(97)$ & 0.66 \\
\hline $2(3)$ & \\
\hline TAVI-GA $(n=74)$ & \\
\hline $72(97)$ & 0.91 \\
\hline $2(3)$ & \\
\hline
\end{tabular}

